\title{
My experience from ECOSEP Bauerfeind Travelling Fellowship 2013
}

\author{
Malliaropoulos Nikos ${ }^{1}$ \\ Xavier Valle ${ }^{2}$ \\ Kyriakos Tsapralis ${ }^{3}$ \\ Heinz Lohrer ${ }^{4}$ \\ Nat Padhiar ${ }^{5}$ \\ Nicola Maffulli 6
}

1 National Track \& Field Centre, Sports Injury Clinic, Sports Medicine Clinic of SEGAS, Thessaloniki, Greece

2 Medical Services F.C. Barcelona; High Performance Center (CAR de Sant Cugat - Consorci Sanitari de Terrassa), Barcelona, Spain

3 Isokinetic Medical Group, Bologna, Italy

4 Institute for Sports Medicine, Otto-Fleck-Schneise 10, Frankfurt am Main, Germany; Institute for Sports and Sports Science, Albert-Ludwigs-University Freiburg i.Brsg., Germany

5 John King Centre for Leg Pain, London Independent Hospital, London, UK

6 Head of Department of Physical and Rehabilitation Medicine, University of Salerno, Azienda Ospedaliera San Giovanni Di Dio e Ruggi d'Aragona, Salerno, Italy Centre for Sports and Exercise Medicine, Queen Mary University of London, Barts and The London School of Medicine and Dentistry, Mile End Hospital, London, UK

I am George Bikos resident in Physical Medicine and Rehabilitation in Athens and Associated member of the European College of Sports and Exercise PhysiciansECOSEP with interest in musculoskeletal medicine and therefore I applied for this fellowship. I was selected to participate in ECOSEP Travelling Fellowship 2013 sponsored by Bauerfeind and ECOSEP between May and September 2013. The program included visiting five Sports medicine centres across Europe and spending 1 week in each centre. The objective of the fellowship was to experience how a sport and exercise physician addresses pathological musculoskeletal conditions, to record the diagnostic and treatment algorithms was used in certain situations and finally to detect any basic differences and similarities among sport centres. The centres were: National Track and Field Center, Sports Medicine Clinic of SEGAS in Thessaloniki, Greece, the Sports and Exercise Medicine Institute in Frankfurt, Germany, the Isokinetic Medical Centre in Bologna, Italy, the FC Barcelona medical center and the CAR High Performance Sports Centre in Barcelona, Spain and finally the BMI Independent Hospital in cooperation with Centre of SEM in Barts and the London School of Medicine \& Dentistry in England.
I attended numerous outpatient clinics, therapeutics interventions, interesting research protocols, athletes' screening tests and some surgeries. Despite, there were an expected slight variation in treatment approaches among centres, preference in some centres to homeopathic remedies or variated extent of the multi-disciplinary sport team, I noticed many basic similarities:

- All of them are multidisciplinary organizations composed from Sport and Exercise Physician, Sports Orthopedic Surgeons, Physiotherapists, and Exercise physiologists.

- Additionally all of them had research department for studies related to exercise physiology, sport injuries, new effectiveness of several tests.

- History and clinical examination were followed by diagnostic ultrasound, as a primary diagnostic tool in all centers. And it was used also to guide treatment with injections.

- There was a common treatment rationale of using 'exercise prescription' for treatment of common musculoskeletal conditions.

Regarding my personal impact ECOSEP Bauerfeind Travelling Fellowship was certainly a life-changing experience.

I had the chance to visit in a short period of time some of most renowned sport rehabilitation centres and to make new friends and connections. I experienced the new trends in Sports and Exercise Medicine and how they manage several musculoskeletal conditions across Europe. Sport and Exercise Medicine (SEM) is a relatively new medical specialty and therefore there is a need for establishing interventions based on evidencebased principles. Additionally treatment from SEM Physician is not only useful for elite athletes but it can be beneficiary for a wide range of acute and chronic musculoskeletal conditions in common population.

According to my opinion, this makes imperative the establishment of SEM as an independent medical specialty across Europe, including my country Greece.

Additionally I would strongly support as a resident in Physical Medicine and Rehabilitation (PRM) that SEM physician could be part of a multidisciplinary team in a PRM unit for neurologic patients with co-existing musculoskeletal morbidities using expertise in exercise prescription, biomechanics, gait analysis, nutrition, etc. In conclusion, after this didactic European tour, I realize the great potential of Sport and Exercise Medicine as a medical specialty and also that by standardization of therapeutic interventions, through collaboration and dispersion of knowledge, Sport and Exercise Medicine has a very promising future.

George Bikos 